\title{
Effects of Dietary Xanthophylls, Canthaxanthin and Astaxanthin on $N$-Methyl- $N$-nitrosourea-induced Rat Mammary Carcinogenesis
}

\author{
TAKASHI YURI, KATSUHIKO YOSHIZAWA, YUKO EMOTO, \\ YUICHI KINOSHITA, MICHIKO YUKI and AIRO TSUBURA \\ Department of Pathology 2, Kansai Medical University, Hirakata, Japan
}

\begin{abstract}
Background: Natural xanthophylls, canthaxanthin and astaxanthin are known to exhibit anticancer activity. However, the dietary effects of canthaxanthin and astaxanthin on $N$-methyl-N-nitrosourea (MNU)-induced mammary cancer remain controversial, and their mechanisms of action have not been clearly identified. Materials and Methods: Threeweek-old female Sprague-Dawley rats were fed a xanthophyll-free (basal diet) diet or experimental diets containing canthaxanthin or astaxanthin $(0.04 \%$ and $0.4 \%)$ for 5 weeks (until 8 weeks of age), after which all rats were provided the basal diet ( $n=15$ each). Rats were administered $M N U$ at 6 weeks of age, and the incidence of mammary tumors at 20 weeks of age was compared. The expression of adiponectin in mammary adipose tissues taken at 7 weeks of age was also compared. Results: Compared to the basal diet group, the $0.4 \%$ (but not the 0.04\%) astaxanthin diet significantly reduced the incidence of palpable mammary carcinoma $(92 \%$ vs. $42 \%$; $p<0.05)$, while the low and high canthaxanthin diets produced no significant inhibition. Adiponectin immunoblotting showed significantly higher expression in the $0.4 \%$ astaxanthin diet group, while the other groups were similar to the basal diet group. Conclusion: High concentrations of astaxanthin suppress $M N U$-induced mammary carcinoma. Changes in adiponectin may be involved in the mechanism of action.
\end{abstract}

Dietary factors play a crucial role in modulating the development and growth of certain types of cancer. The chemopreventive actions of natural compounds including xanthophylls are thought to be attributable to their antioxidant

Correspondence to: Takashi Yuri, Department of Pathology 2, Kansai Medical University, Hirakata, Osaka, 573-1010, Japan. Tel.: +81 728042373, e-mail: yurit@ hirakata.kmu.ac.jp

Key Words: Xanthophyll, canthaxanthin, astaxanthin, adiponectin, mammary cancer. properties (1). Canthaxanthin $(4,4$ '-diketo- $\beta$-caroten) is a natural marine xanthophyll that has been approved for use as a colorant of foods, beverages and pharmaceutical preparations (2). Astaxanthin (3,3'-dihydroxy- $\beta, \beta$-caroten4,4 '-dione) is another xanthophyll that is widely distributed in the red pigment of shrimp and salmon and has been approved as a food additive for use in aquacultural industries (3). These natural xanthophylls are considered powerful antioxidative and anti-inflammatory nutrients (4), and have potential for improving insulin-resistant status (5). Biological activities related to the anticancer properties of xanthophylls have also been repeatedly described (6-8). However, the precise anticarcinogenic mechanisms of these xanthophylls remain unclear, especially when administered during early stages of carcinogenesis.

In this study, we investigated whether short-term dietary xanthophyll administration (canthaxanthin and astaxanthin) inhibits $N$-methyl- $N$-nitrosourea (MNU)-induced rat mammary carcinogenesis during initiation until early promotional stages of cancer development. Additionally, changes in adipocytokine production in mammary adipose tissues of xanthophyll-administered rats were examined in order to elucidate their mechanism of action.

\section{Materials and Methods}

Animals and experimental procedures. Ninety female SpragueDawley rats (aged 3 weeks) were purchased from Charles River Japan (Hino, Japan). The rats were randomly divided into five groups (xanthophyll-free basal diet; 0.04 and $0.4 \%$ canthaxanthin; and 0.04 and $0.4 \%$ astaxanthin diet) and housed at a maximum of five rats per cage in an environmentally controlled animal room at our university facility. Each of the 18 rats were either fed a basal diet (AIN-76A, Oriental Yeast, Tokyo, Japan) or xanthophyllcontaining diets with either 0.04 or $0.4 \%$ canthaxanthin (LKT Laboratories Inc., St. Paul, MN, USA), or 0.04 or $0.4 \%$ astaxanthin (Sequoia Research, Pangbourne, Berkshire, UK) for 5 weeks starting at 3 weeks of age. Subsequent to the treatment period, the rats were switched to a basal (AIN-76A) diet until the end of the experiment (at 20 weeks of age). Body weight changes were 
Table I. Diet and xanthophyll intake and body weight at 3 and 8 weeks of age in Sprague-Dawley rats.

\begin{tabular}{lcccc}
\hline Group & $\begin{array}{c}\text { Daily intake of diet } \\
(\mathrm{g})(\mathrm{n}=18)\end{array}$ & $\begin{array}{c}\text { Daily intake of xanthophylls } \\
(\mathrm{mg})(\mathrm{n}=18)\end{array}$ & $\begin{array}{c}\text { Body weight at } 3 \text { weeks of age } \\
(\mathrm{g})(\mathrm{n}=18)\end{array}$ & $\begin{array}{c}\text { Body weight at } 8 \text { weeks of age } \\
(\mathrm{g})(\mathrm{n}=15)\end{array}$ \\
\hline Basal diet & $9.8 \pm 0.4$ & 0 & $43.8 \pm 0.8$ & $187.8 \pm 1.7$ \\
$0.04 \% \mathrm{Cx}$ & $10.1 \pm 0.8$ & $3.9 \pm 0.3$ & $44.5 \pm 0.5$ & $192.5 \pm 1.7$ \\
$0.4 \% \mathrm{Cx}$ & $10.5 \pm 0.5$ & $41.8 \pm 0.7$ & $43.8 \pm 1.8$ & $188.1 \pm 2.1$ \\
$0.04 \% \mathrm{Ax}$ & $10.5 \pm 0.2$ & $4.2 \pm 0.5$ & $43.7 \pm 0.5$ & $191.5 \pm 1.5$ \\
$0.4 \% \mathrm{Ax}$ & $11.4 \pm 0.2$ & $45.5 \pm 0.6$ & $46.2 \pm 0.9$ & $194.1 \pm 2.0$ \\
\hline
\end{tabular}

Cx: Canthaxanthin, Ax: astaxanthin. No. of animals used at 3 and 8 weeks of age was 18 (without $N$-methyl- $N$-nitrosourea) and 15 (with $N$-methyl$N$-nitrosourea) rats per group, respectively.

recorded throughout the experiment. At 6 weeks of age, 15 rats per group were intraperitoneally injected with $60 \mathrm{mg} / \mathrm{kg}$ MNU (Sigma, St. Louis, MO, USA) for the induction of mammary cancer. The remaining rats (three rats per group without MNU administration) were sacrificed at 7 weeks of age, and bilateral abdominal mammary adipose tissue (including mammary gland) was used for immunoblotting analysis and immunohistological examination. In MNU-treated rats, moribund animals were sacrificed and excluded from the experiment.

The incidence of palpable mammary tumors (largest tumor diameter $>1 \mathrm{~cm}$ ) were recorded weekly, and 14 weeks after MNU injection (at 20 weeks of age) all surviving rats were sacrificed and palpable mammary tumors were surgically resected for histological and immunohistological examinations. All animal procedures were approved by the Animal Experimentation Committee of Kansai Medical University (certification number: 15-104).

Electrophoresis and western blotting. Adiponectin expression in mammary adipose tissues obtained from non-MNU-treated 7-weekold rats was investigated. Unilateral abdominal mammary tissues were cut into small pieces and washed with PBS(-). Fresh samples were homogenized with PRO-PREP protein extraction solution (iNtRON Biotechnology, Sungnam, South Korea). Protein concentrations were measured using the DC protein assay kit (BioRad, Hercules, CA, USA). Proteins from each sample were electrophoresed on poly-acrylamide gels and electroblotted onto transfer membranes (Hybond-P PVDF membranes; Amersham Biosciences, Buckinghamshire, UK). The transfer membranes were incubated with anti-adiponectin C-terminal antibody (ab181699; Abcam, Cambridge, UK) overnight followed by incubation with secondary antibody (anti-rabbit $\mathrm{IgG}$, horseradish peroxidase-linked antibody; Cell Signaling Technology, Danvers, MA, USA). Protein bands were visualized using ECL plus reagent (Amersham Biosciences) and analyzed with a lumino-image analyzer (LAS4000 mini; Fuji Film, Tokyo, Japan). The relative intensity of the protein bands was compared against the intensity of the loading control ( $\beta$-actin, AC-15; Abcam). Values of relative intensities per group were calculated from three independent samples.

Histological and immunohistochemical examinations. Contralateral abdominal mammary tissues were surgically resected from nonMNU-treated 7-week-old rats. Mammary tissues were fixed with $10 \%$ neutral buffered formalin, embedded in paraffin, cut into $4 \mu \mathrm{m}$ sections, and stained with hematoxylin and eosin for routine histological examination. Palpable mammary tumors were examined histologically and were confirmed as carcinoma. Expression of adiponectin receptor 1 and 2 (ADIPO R-1 and R-2; H-40 and H-44, dilution 1:50; Santa Cruz Biotechnology, Dallas, TX, USA) in mammary glands and MNU-induced mammary carcinomas were evaluated. The immunohistochemical procedure is described in detail elsewhere (9). The expression levels of Adipo R-1 and R-2 in mammary carcinomas were graded on a scale of 0 to 2 based on the staining intensities and proportions of cytoplasm-reactive cancer cells. In brief, the expression levels were scored 0 if no cytoplasmreactive cancer cells were detected, 1 if staining was weakly positive in $<1 / 3$ of cancer cells, and 2 if staining was weakly positive in $>2 / 3$ of cells or strongly positive in $>1 / 3$ of cancer cells (10). The expression levels in mammary cancers in the basal diet group and the $0.4 \%$ astaxanthin diet group $(n=11$ and $n=5$, respectively; randomly selected from the largest sized mammary carcinoma in tumor-bearing rats) were compared.

Statistical analysis. All data are expressed as the mean \pm standard error. Data were first assessed for homogeneity of variance and then evaluated using the unpaired $t$-test or Mann-Whitney $U$-test. Probability values of $p<0.05$ were considered statistically significant.

\section{Results}

Food intake and body weight changes. At the end of the xanthophyll diet feeding period (at 8 weeks of age), the daily intake of diets containing $0,0.04$ or $0.4 \%$ xanthophyll (basal diet, low and high canthaxanthin or astaxanthin) were similar among groups and body weights were similar among groups at both 3 and 8 weeks of age (Table I). Daily dietary xanthophyll intake was determined to be approximately $4 \mathrm{mg}$ and $>40 \mathrm{mg}$ in the $0.04 \%$ and $0.4 \%$ xanthophyll-containing diets, respectively. The xanthophyll doses used in our study were not toxic to female Sprague-Dawley rats.

Effects of dietary xanthophylls on mammary carcinogenesis. At 6 weeks of age, 15 rats per group were intraperitoneally injected with $60 \mathrm{mg} / \mathrm{kg} \mathrm{MNU}$ for mammary carcinoma induction. In our protocol, experimental diets containing xanthophylls started at 3 weeks of age (after weaning) and 


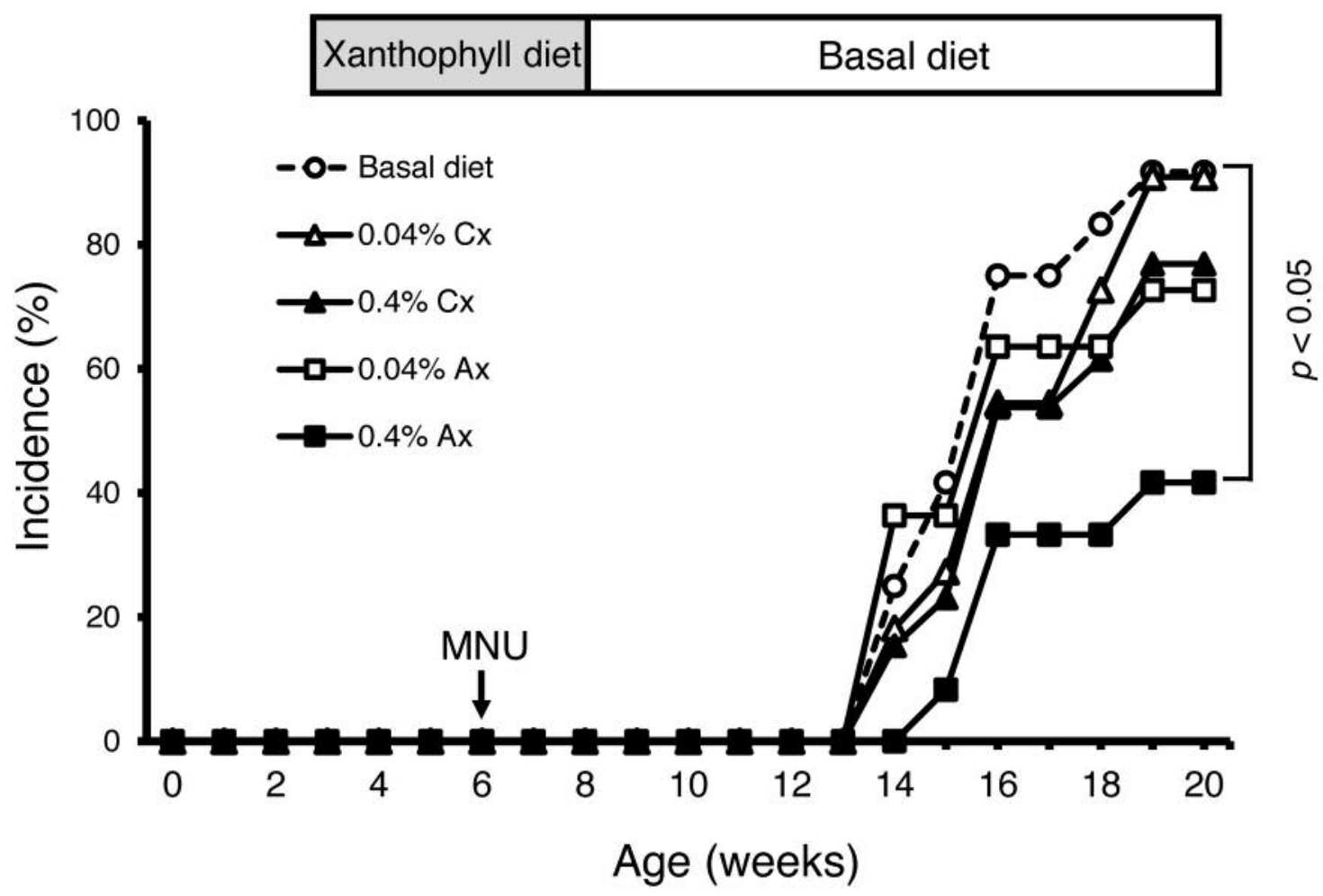

Figure 1. Effects of xanthophyll diets on the cumulative incidence of $N$-methyl-N-nitorosourea-induced mammary carcinoma in female SpragueDawley rats. Cx: Canthaxanthin; Ax: astaxanthin.

continued for 5 weeks. Thus, the ability of dietary xanthophylls to influence the initiation and early promotional stages of carcinogenesis was examined. During the course of the experiment, moribund rats (2-4 rats per each group) were sacrificed and excluded from the study. Only healthy rats surviving until the end of the experiment (20 weeks of age) were included in the study. All animals developed mammary tumors, that were diagnosed histologically as papillary or cribriform carcinoma. The cumulative incidence of palpable mammary carcinoma is shown in Figure 1. The palpable mammary tumors were first detected 8 weeks after MNU injection (at 14 weeks of age). Thereafter, the increase in the cumulative incidence of palpable mammary carcinomas in the $0.4 \%$ astaxanthin group were comparatively lower than those of the other groups. Lastly, at the termination of the experiment (14 weeks after MNU, 20 weeks of age), the incidence of palpable mammary tumors in the $0.4 \%$ astaxanthin group was significantly lower compared to the basal diet group (Figure 1 and Table II; $42 \%$ vs. 92\%). Although the latency was approximately equal among the groups, tumor multiplicity (numbers of palpable mammary carcinoma per rat) and mean carcinoma wet weight were lower in the $0.4 \%$ astaxanthin group when compared to the other groups; however, the differences did not reach statistical significance.
Changes in adiponectin expression in mammary adipose tissues. Next, adiponectin expression in mammary adipocytes from 7-week-old rats (rats fed experimental diets for 4 weeks) was compared using immunoblotting. Representative adiponectin expression and relative band intensities from three samples each are shown in Figure 2A and $\mathrm{B}$, respectively. The band intensities in samples from the basal diet, low canthaxanthin diet and high canthaxanthin diet groups were almost the same. Conversely, the expression levels in the $0.04 \%$ astaxanthin group were slightly increased, and the relative band intensity in the $0.4 \%$ astaxanthin group was significantly higher compared to the basal diet group $(p=0.011)$.

Expression of ADIPO $R-1$ and $R-2$ in mammary gland and mammary carcinomas. Immunohistochemical analysis of ADIPO R-1 and R-2 expression was conducted to assess adiponectin receptor expression in normal mammary glands and mammary carcinomas from basal and $0.4 \%$ astaxanthin diet fed rats. ADIPO R-1 and R-2 were simultaneously expressed in the cytoplasm of mammary epithelial cells harvested at 7 weeks of age (Figure 3). ADIPO R-2 expression was relatively weaker than ADIPO R-1 expression. The intensities of expression were quantitatively 
Table II. $N$-methyl-N-nitrosourea-induced rat mammary carcinoma in relation to xanthophyll diet.

\begin{tabular}{lccccccc}
\hline Group & $\begin{array}{c}\text { Initial no. } \\
\text { of rats }\end{array}$ & $\begin{array}{c}\text { Effective no. } \\
\text { of rats }\end{array}$ & $\begin{array}{c}\text { Body weight at } \\
\text { 20 weeks of age }(\mathrm{g})\end{array}$ & $\begin{array}{c}\text { Incidence of }>1 \mathrm{~cm} \\
\text { carcinoma (\%) }\end{array}$ & $\begin{array}{c}\text { Latency } \\
(\mathrm{days})^{\mathrm{a}}\end{array}$ & $\begin{array}{c}\text { Multiplicityb } \\
\text { Mean wet weight } \\
\text { of carcinoma (g) }\end{array}$ \\
\hline Basal diet & 15 & 12 & $272.1 \pm 7.8$ & 92 & $68 \pm 3$ & $3.3 \pm 0.5$ & $3.9 \pm 0.6$ \\
$0.04 \% \mathrm{Cx}$ & 15 & 11 & $290.0 \pm 5.8$ & 91 & $74 \pm 4$ & $3.8 \pm 0.7$ & $4.3 \pm 1.0$ \\
$0.4 \% \mathrm{Cx}$ & 15 & 13 & $279.1 \pm 10.1$ & 77 & $72 \pm 4$ & $2.4 \pm 0.3$ & $3.5 \pm 0.4$ \\
$0.04 \% \mathrm{Ax}$ & 15 & 11 & $269.4 \pm 5.9$ & 73 & $66 \pm 4$ & $2.6 \pm 0.4$ & $3.4 \pm 0.4$ \\
$0.4 \% \mathrm{Ax}$ & 15 & 12 & $274.7 \pm 5.6$ & $42^{*}$ & $73 \pm 5$ & $1.8 \pm 0.3$ & $2.8 \pm 0.8$ \\
\hline
\end{tabular}

Cx: Canthaxanthin, Ax: astaxanthin. a Duration between carcinogen injection to first detection of $>1 \mathrm{~cm}$ mammary tumor. ${ }^{\mathrm{b}}$ Numbers of mammary carcinomas $>1 \mathrm{~cm}$ per rats. ${ }^{*} p<0.05$ Compared to the basal diet group.

evaluated in mammary carcinoma at 20 weeks of age. ADIPO R-1 and R-2 were expressed in mammary carcinomas, and the expression scores were relatively higher in the $0.4 \%$ astaxanthin group $(\mathrm{R} 1=1.5 \pm 0.2, \mathrm{R} 2=1.3 \pm 0.3$ ) when compared with the basal diet group ( $\mathrm{R} 1=1.2 \pm 0.3$, $\mathrm{R} 2=0.9 \pm 0.1)$; however, the differences did not reach significance ( $p=0.11$ and 0.12 , respectively).

\section{Discussion}

The present study showed that oral astaxanthin intake during the early developmental stage suppressed MNU-induced rat mammary carcinogenesis. In our protocol, the administration of experimental diets containing xanthophylls at different concentrations was initiated at 3 weeks of age (after weaning) and continued for 5 weeks (until 8 weeks of age), and the carcinogenic stimuli was given at 6 weeks of age. Therefore, xanthophylls were provided during the initiation and early promotional stages of mammary carcinogenesis. Canthaxanthin and astaxanthin have been detected physiologically in human breast tissue, and the amount is inversely related to the frequency of malignant lesions (11). In agreement with our present protocol, the inhibitory effects on mouse fibrosarcoma cells were evident only when astaxanthin intake was started 1 or 3 weeks before transplantation (12), and oral astaxanthin intake at the preinitiation stage reduced the incidence of 1,2-dimethylhydrazineinduced rat colon aberrant crypt foci and crypt cell proliferation more significantly than intake during the post-initiation stage (13). Accordingly, the present study demonstrated that a potent anticancer effect was observed in rats fed a diet containing $0.4 \%$ astaxanthin. Tumor incidence was significantly suppressed, and tumor multiplicity and mean carcinoma weight tended to be suppressed. However, daily administration of $50 \mathrm{mg} / \mathrm{kg}$ body weight astaxanthin did not reduce the incidence of MNUinduced mammary carcinoma in Wistar rats (14). In agreement, $0.4 \%$ astaxanthin in the diet, but not $0.04 \%$ astaxanthin, may play a crucial role in mammary carcinogenesis when administered during the pre-initiation stage.

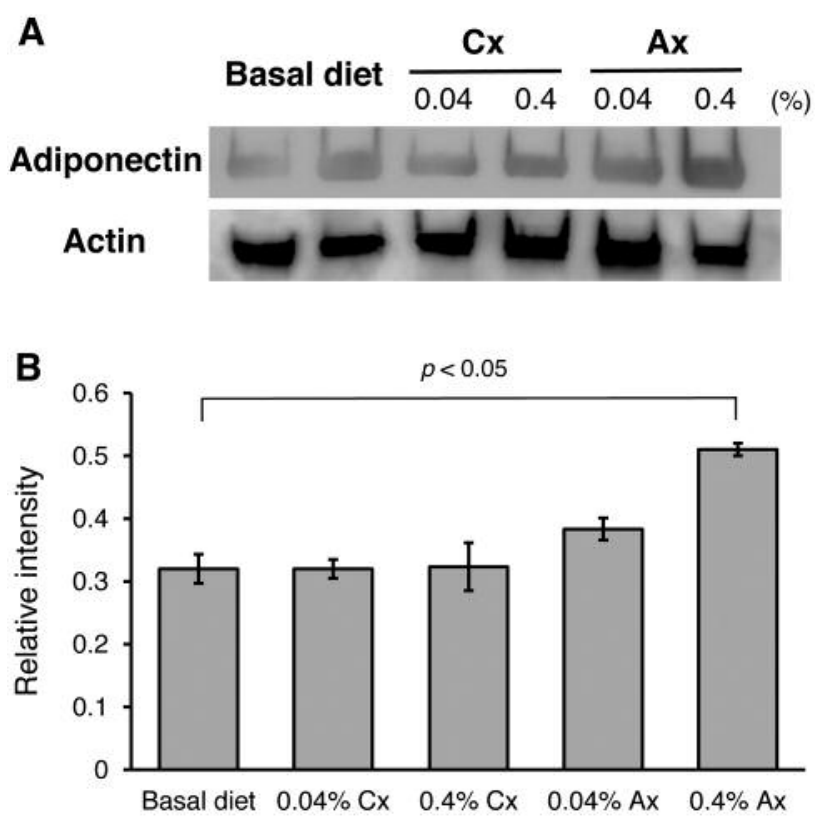

Figure 2. Changes in adiponectin expression in mammary adipose tissues from 7-week-old female Sprague-Dawley rats fed xanthophyll diets for 4 weeks. A: Representative immunoblotting results. B: Relative band intensities of adiponectin. Cx: Canthaxanthin; Ax: astaxanthin.

Dietary supplementation with canthaxanthin reduces the number of 7,12- dimethylbenz $[\alpha]$ anthracene-induced mammary tumors (7). However, when comparing the anticancer potential of canthaxanthin and astaxanthin, the growth inhibitory action of astaxanthin was observed to be greater than canthaxanthin in a mouse mammary tumor inoculation assay (8). This is in agreement with the present observation that the protective effects of canthaxanthin against mammary cancer may be weaker than astaxanthin in rats.

Several mechanisms underlying the anticancer properties of xanthophylls have been identified. One of the mechanisms is 
Mammary gland (7 weeks of age)

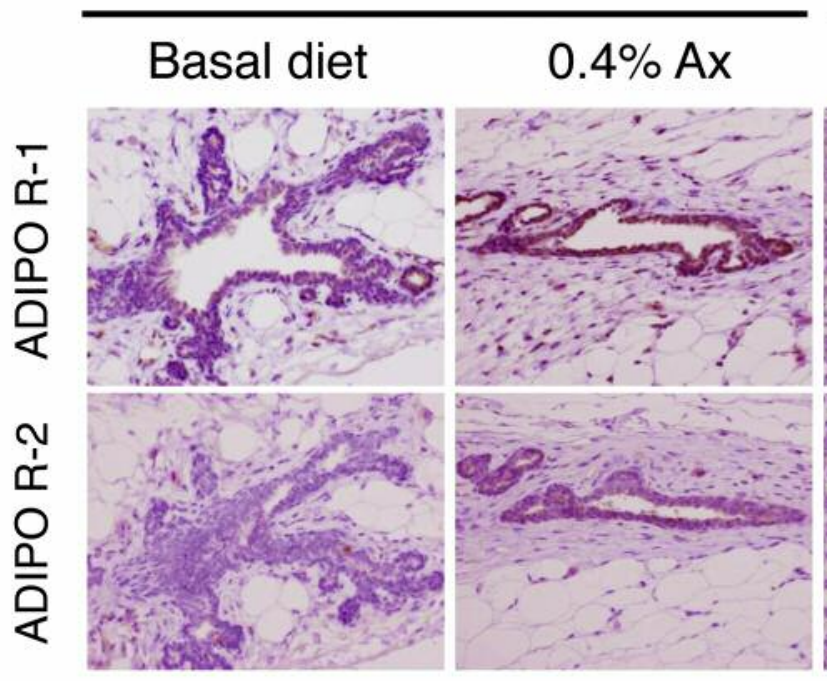

\section{Mammary carcinoma (20 weeks of age)}

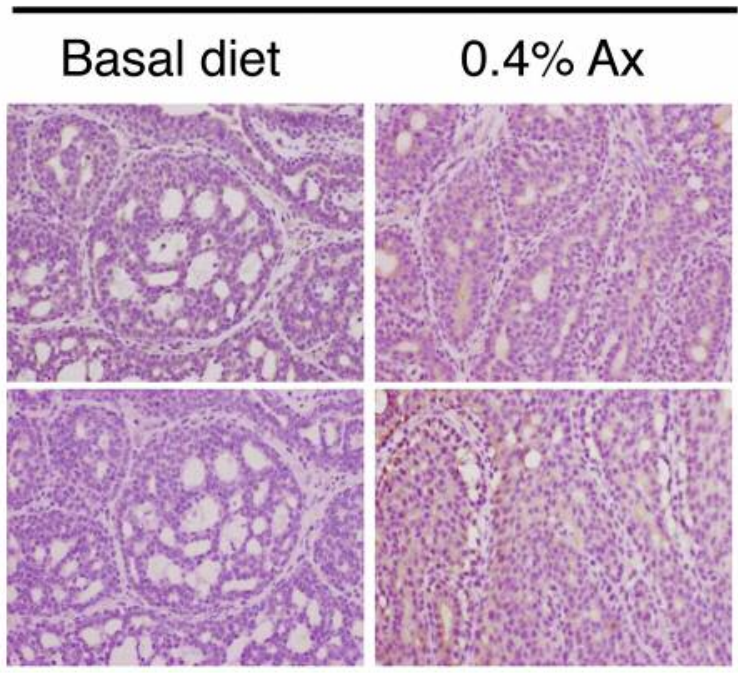

Figure 3. Adiponectin receptor-1 and 2 (ADIPO R-1 and R-2) expression in mammary glands and carcinoma induced by $N$-methyl-N-nitorosourea in female Sprague-Dawley rats. Representative immunohistochemical results. Ax: Astaxanthin.

due to the reduction of oxidative stress and activation of immune responses in host animals. Dietary astaxanthin reduces serum oxidative stress and increases antioxidant enzymes, such as glutathione peroxidase 1 , in mouse white adipose tissues $(13,15)$. Dietary intake of $0.05 \%$ astaxanthin for 8 weeks was found to suppress the development and growth of inoculated mouse mammary tumor cells due to an increased population of plasma natural killer cells and elevated interferon- $\gamma$ levels (16). Moreover, astaxanthin intake protects against stress-induced metastasis of mouse cutaneous mastocytoma cells by blocking hepatic lipid peroxidation (17).

Other known anticancer mechanisms of xanthophylls are due to alterations in cell kinetics, such as inducing programmed cell death or cell-cycle arrest. Astaxanthin supplementation induced mitochondria-mediated cell death in hepatocellular carcinoma cells by up-regulating apoptosisrelated proteins (18). Modulation of cell-cycle progression and apoptosis-related proteins by astaxanthin has been observed in human colon carcinoma cells (19). Furthermore, dietary intake of astaxanthin inhibited the proliferation of rat oral squamous epithelial cells (20).

Increases in serum adiponectin are observed in hyperlipidemic patients (21), which can improve insulin sensitivity in rats with metabolic syndrome (22). Adiponectin is an adipocyte-secreted hormone that may be involved in mediating anticancer actions. Since adiponectin is known to reduce cyclin D1 expression by blocking phosphorylation of glycogen synthase kinase-3 $\beta$ (23), the protective effect of astaxanthin against mammary carcinogenesis may be associated with increased adiponectin levels. Immunoblotting results showed increased expression of adiponectin in mammary tissues in rats fed $0.4 \%$ astaxanthin diet compared to rats fed the basal diet. These results suggest that the inhibitory action of astaxanthin on mammary carcinogenesis appears to be partly due to enhanced adiponectin secretion from adipocytes. In human breast cancer samples, high adiponectin expression is inversely correlated with tumor stage (24). In addition, immunohistochemistry revealed that both ADIPO R-1 and R-2 expression was observed in all mammary carcinomas, and although not significantly, stronger expression was found in tumors of rats fed the astaxanthin diet than in those fed the basal diet. Based on clinical observations, we hypothesize that high expression of adiponectin in mammary tissues of rats fed $0.4 \%$ astaxanthin may be associated with the development of ADIPO R-1 and $\mathrm{R}$-2-positive mammary carcinoma, which are regarded to confer better prognosis for patients with breast cancer (24). Thus, enhanced activation of the adiponectin pathway may suppress mammary carcinogenesis.

In conclusion, dietary administration of $0.4 \%$ astaxanthin during the initiation and early promotion stages significantly suppressed rat mammary carcinogenesis. In contrast, inhibitory effects of $0.04 \%$ and $0.4 \%$ canthaxanthin and $0.04 \%$ astaxanthin were not observed. The immunoblotting and immunohistochemical results indicated that a possible mechanism explaining the effects of astaxanthin might be related to the secretion of adiponectin from mammary adipocytes. However, further studies are required for a complete understanding of the mechanisms underlying the ability of astaxanthin to lower the risk for breast cancer. 


\section{Acknowledgements}

This work was supported by a Grant-in-Aid for Scientific Research (C) (15K00859) from the Japan Society for the Promotion of Science (to T.Y.).

\section{References}

1 Stavric B: Role of chemopreventers in human diet. Clin Biochem 27: 319-332, 1994.

2 Daun H: The chemistry of carotenoids and their importance in food. Clin Nutr 7: 97-100, 1988.

3 Guerin M, Huntley ME and Olaizora M: Haematococcus astaxanthin: Applications for human health and nutrition. Trends Biothechnol 21: 210-216, 2003.

4 Chan KC, Mong MC and Yin MC: Antioxidative and antiinflammatory neuroprotective effects of astaxanthin and canthaxanthin in nerve growth factor differentiated PC12 cells. J Food Sci 74: H225-231, 2009.

5 Ni Y, Nagashimada Y, Zhuge F, Zhan L, Nagata N, Tsutsui A, Nakanuma Y, Kaneko S and Ota T: Astaxanthin prevents and reverses diet-induced insulin resistance and steatohepatitis in mice: A comparison with vitamin E. Sci Rep 5: 17192 DOI: 10.1038/srep17192, 2005.

6 Grubbs CJ, Eto I, Juliana M and Whitaker LM: Effect of canthaxanthin on chemically induced mammary carcinogenesis. Oncology 48: 239-245, 1991.

7 Chew BP, Park JS, Wong MW and Wong T: A comparison of the anticancer activities of dietary $\beta$-carotene, canthaxanthin and astaxanthin in mice in vivo. Anticancer Res 19: 1849-1854, 1999.

8 Zhang L and Wang H: Multiple mechanisms of anticancer effects exerted by astaxanthin. Mar Drugs 13: 4310-4330, 2015.

9 Yuri T, Lai YC, Yoshizawa K and Tsubura A: Human chorionic gonadotropin inhibits N-methyl-N-nitrosourea-induced mammary carcinoma growth in female Lewis rats. In Vivo 26: 361-368, 2012.

10 Jeong YJ, Bong JG, Park SH, Choi JH and Oh HK: Expression of leptin, leptin receptor, adiponectin, and adiponectin receptor in ductal carcinoma in situ and invasive breast cancer. J Breast Cancer 14: 96-103, 2011.

11 Czeczuga-Semeiniuk E and Wolczynski S: Does variability in carotenoid composition and concentration in tissue of the breast and reproductive tract in women depend on type of lesion? Adv Med Sci 53: 270-277, 2008

12 Jyonouchi H, Sun S, Iijima K and Gross MD: Antitumor activity of astaxanthin and its mode of action. Nutr Cancer 36: 59-65, 2000.

13 Prabhu PN, Ashokkumar P and Sudhandiran G: Antioxidative and antiproliferative effects of astaxanthin during the initiation stages of 1,2-dimethylhydrazine-induced experimental colon carcinogenesis. Fundam Clin Pharmacol 23: 225-234, 2009.
14 Andrei S, Cernea C, Taulescu M and Catoi M: Effects of astaxanthin supplementation on chemically induced tumorigenesis in Wistar rats. Acta Vet Scand 54(50): doi: 10.1186/1751-0147-54-50, 2012.

15 Ohno T, Shimizu M, Shirakami Y, Miyazaki T, Ideta T, Kochi T, Kubota M, Sakai H, Tanaka T and Moriwaki H: Preventive effects of astaxanthin on diethylnitrosamine-induced liver tumorigenesis in C57/BL/Ksj-db/db obese mice. Hepatol Res 46: E201-209, 2016.

16 Nakao R, Nelson OL, Park JS, Mathison BD, Thompson PA and Chew BP: Effect of dietary astaxanthin at different stages of mammary tumor initiation in $\mathrm{BALB} / \mathrm{c}$ mice. Anticancer Res 30 : 2171-2176, 2010.

17 Kurihara H, Koda H, Asami S, Kiso $\mathrm{Y}$ and Tanaka T: Contribution of the antioxidative property of astaxanthin to its protective effect on the promotion of cancer metastasis in mice treated with restraint stress. Life Sci 70: 2509-2520, 2002.

18 Song XD, Zhang JJ, Wang MR, Liu WB, Gu XB and Lv CJ: Astaxanthin induces mitochondria-mediated apoptosis in rat hepatocellular carcinoma CBRH-7919 cells. Biol Pharm Bull 34: 839-844, 2011

19 Palozza P, Torelli C, Boninsegna A, Simone R, Catalano A, Mele MC and Picci N: Growth-inhibitory effects of the astaxanthinrich alga Haematococcus pluvialis in human colon cancer cell. Cancer Lett 283: 108-117, 2009.

20 Tanaka T, Makita H, Ohnishi M, Mori H, Satoh K and Hara A: Chemoprevention of rat oral carcinogenesis by naturally occuring xanthophils, astaxanthin and canthaxanthin. Cancer Res 55: 4059-4064, 1995.

21 Yoshida H, Yanai H, Ito K, Tomono Y, Koikeda T, Tsukahara H and Tada N: Administration of natural astaxanthin increases serum HDL-cholesterol and adiponectin in subjects with mild hyperlipidemia. Atherosclerosis 209: 520-523, 2010.

22 Hussein G, Nakagawa T, Goto H, Shimada Y, Matsumoto K, Sankawa $U$ and Watanabe $\mathrm{H}$ : Astaxanthin ameliorates features of metabolic syndrome in SHR/NDmcr-cp. Life Sci 80: 522-529, 2007.

23 Wang Y, Lam JB, Lam KSL, Liu J, Lam MC, Hoo RLC, Wu D, Cooper GJS and $\mathrm{Xu}$ A: Adiponectin modulates the glycogen synthase kinase-3 $/ \beta / \beta$-catenin signaling pathway and attenuates mammary tumorigenesis of MDA-MB-231 cells in nude mice. Cancer Res 66: 11462-11470, 2006.

24 Sonmez B, Seker M, Bilici A, Yavuz Erkal F, Oven Ustaalioglu BB, Gumus M, Ozturk Guler D, Karaduman M, Gezen C, Eser $\mathrm{M}$, Bildik $\mathrm{N}$ and Salepci T: Is there any correlation among adiponectin levels in serum, tumor tissue and normal tissue of the same patients with breast cancer? J BUON 16: 227-232, 2011.

Received July 21, 2016

Revised August 4, 2016

Accepted August 5, 2016 